\title{
Relative profit maximization and Bertrand equilibrium with quadratic cost functions
}

\author{
Atsuhiro Satoh ${ }^{1} \cdot$ Yasuhito Tanaka $^{2 *}$ \\ ${ }^{1}$ Faculty of Economics, Osaka University of Commerce, Osaka, Japan \\ ${ }^{2}$ Faculty of Economics, Doshisha University, Kyoto, Japan
}

Received: 23 July 2013

Revised: 22 September 2013

Accepted: 23 September 2013

\begin{abstract}
We study the Bertrand equilibrium in duopoly in which two firms produce a homogeneous good under quadratic cost functions, and they seek to maximize the weighted sum of their absolute and relative profits. We show that there exists a range of the equilibrium prices in duopolistic equilibria. This range of equilibrium prices is narrower and lower than the range of the equilibrium prices in duopolistic equilibria under pure absolute profit maximization, and the larger the weight on the relative profit, the narrower and lower the range of the equilibrium prices. In this sense relative profit maximization is more aggressive than absolute profit maximization.
\end{abstract}

Keywords: Bertrand equilibrium, quadratic cost function, relative profit maximization JEL Classification Codes: D43, L13

\section{Introduction}

We study the Bertrand equilibrium in duopoly in which two firms produce a homogeneous good under quadratic cost functions, and they seek to maximize the weighted sum of their absolute and relative profits instead of their absolute profits themselves. The relative profit of a firm is the difference between its absolute profit and the absolute profit of the rival firm.

In recent years, maximizing relative profit instead of absolute profit has aroused the interest of economists. For analyses of relative profit maximization see Schaffer (1989), Vega-Redondo (1997), Lundgren (1996), Kockesen et. al. (2000), Matsumura, Matsushima and Cato(2013), Gibbons and Murphy (1990) and Lu (2011). In another paper, Tanaka (2013), we have shown that under relative profit maximization the choice of strategic variables is irrelevant to the outcome in

*Corresponding author. E-mail: yasuhito@mail.doshisha.ac.jp.

Citation: Satoh, A. and Tanaka, Y. (2013) Relative profit maximization and Bertrand equilibrium with quadratic cost functions, Economics and Business Letters, 2(3), 134-139. 
duopoly. In that paper we considered a duopoly with differentiated goods. On the other hand, in this paper we assume that the firms produce a homogeneous good under quadratic cost functions, and set their prices.

We show the following results.

1. When the firms seek to maximize their pure absolute profits, there exists no monopolistic equilibrium, which is an equilibrium where only one firm sells the good. And there exists a range of the equilibrium prices in duopolistic equilibria, which are equilibria where both firms set the same price. In duopolistic equilibria the firms may earn positive absolute profits.

2. When the firms seek to maximize the weighted sum of their absolute and relative profits, also there exists no monopolistic equilibrium, and there exists a range of the equilibrium prices in duopolistic equilibria. This range of equilibrium prices is contained in the range of the equilibrium prices in duopolistic equilibria under pure absolute profit maximization, and the range under relative profit maximization is narrower and lower than the range under absolute profit maximization. The larger the weight on the relative profit, the narrower and lower the range of the equilibrium prices. In this sense relative profit maximization is more aggressive than absolute profit maximization. In duopolistic equilibria the firms may earn positive absolute profits.

\section{The model}

There are two firms, A and B. They produce a homogeneous good. The price of the good of Firm A is $p_{A}$ and the price of the good of Firm B is $p_{B} .0<p_{A}<1$ and $0<p_{B}<1$. The outputs of Firm A and B are denoted, respectively, by $q_{A}$ and $q_{B}$. The firms set the prices of their goods, and consumers buy the good from the firm whose price is lower. Let $p=\min \left\{p_{A}, p_{B}\right\}$. Consumers' demand is represented by the following inverse demand function.

$$
p=1-q_{A}-q_{B} \text {. }
$$

If $p_{A}=p_{B}$, each firm acquires a half of the demand, and two firms constitute a duopoly. Thus, if $p_{A}=p_{B}$

$$
q_{A}=q_{B}=\frac{1-p}{2}
$$

On the other hand, if $p_{A}<p_{B}$ (or $p_{B}<p_{A}$ ) Firm A (or Firm B) acquires total demand, and it becomes a monopolist. Thus, if $p_{A}<p_{B}$,

If $p_{B}<p_{A}$,

$$
q_{A}=1-p_{A} \text { and } q_{B}=0 .
$$

The cost functions of Firm A and B are

$$
q_{B}=1-p_{B} \text { and } q_{A}=0 .
$$

where $c>0$.

$$
c_{A}\left(q_{A}\right)=c q_{A}^{2} \text {, and } c_{B}\left(q_{B}\right)=c q_{B}^{2},
$$

If $p_{A}<p_{B}$, the absolute profit of Firm $\mathrm{A}$ is

$$
\pi_{A}^{M}=\left(1-p_{A}\right) p_{A}-c\left(1-p_{A}\right)^{2}=\left(1-p_{A}\right)\left(p_{A}+c p_{A}-c\right) \text {. }
$$

$M$ denotes monopoly. Of course the profit of Firm B is zero. Similarly if $p_{B}<p_{A}$, we have

$$
\pi_{B}^{M}=\left(1-p_{B}\right) p_{B}-c\left(1-p_{B}\right)^{2}=\left(1-p_{B}\right)\left(p_{B}+c p_{B}-c\right) .
$$

The profit of Firm A is zero.

On the other hand, if $p_{A}=p_{B}$, the absolute profits of Firm A and $\mathrm{B}$ are

$$
\pi_{A}^{D}=\pi_{B}^{D}=\frac{1-p}{2} p-c\left(\frac{1-p}{2}\right)^{2}=\left(\frac{1-p}{4}\right)(2 p+c p-c)
$$


$p$ is the common price, and $D$ denotes duopoly.

The objective of Firm A is the weighted sum of its absolute profit and its relative profit. In duopoly it is expressed as follows.

and the objective of Firm $B$ is

$$
\Pi_{A}^{D}=(1-\alpha) \pi_{A}^{D}+\alpha\left(\pi_{A}^{D}-\pi_{B}^{D}\right)=\pi_{A}^{D}-\alpha \pi_{B}^{D},
$$

where

$$
\Pi_{B}^{D}=(1-\alpha) \pi_{B}^{D}+\alpha\left(\pi_{B}^{D}-\pi_{A}^{D}\right)=\pi_{B}^{D}-\alpha \pi_{A}^{D},
$$

Since, at a duopolistic equilibrium $\pi_{A}^{D}=\pi_{B}^{D}$, we have

$$
0<\alpha<1 \text {. }
$$

$$
\Pi_{A}^{D}=\Pi_{B}^{D}=(1-\alpha) \pi_{A}^{D} .
$$

In monopoly the absolute profit of a firm other than the monopolist is zero. Thus, the absolute profit and the relative profit of the monopolist are equal, and the objective of the monopolist is its absolute profit, that is, if Firm A is a monopolist,

and if Firm B is a monopolist,

$$
\Pi_{A}=\pi_{A}^{M},
$$

$$
\Pi_{B}=\pi_{B}^{M} .
$$

Without loss of generality we assume $p_{A} \leq p_{B}$. Call a firm in duopoly a duopolist. From these formulas we obtain the following results.

1. When $p_{A}<p_{B}$ and $p_{A}<\frac{c}{1+c}$ the profit of the monopolist is negative, when $p_{A}<p_{B}$ and $p_{A}=\frac{c}{1+c}$ the profit of the monopolist is zero, and when $p_{A}<p_{B}$ and $p_{A}>\frac{c}{1+c}$ the profit of the monopolist is positive.

2. When $p_{A}=p_{B}$ and $p_{A}<\frac{c}{2+c}$ the profit of each duopolist is negative, when $p_{A}=p_{B}$ and $p_{A}=\frac{c}{2+c}$ the profit of each duopolist is zero, and when $p_{A}=p_{B}$ and $p_{A}>\frac{c}{2+c}$ the profit of each duopolist is positive.

3. When $p_{A}<\frac{3 c}{2+3 c}$ the profit of the monopolist which is the profit of Firm $\mathrm{A}$ if $p_{A}<p_{B}$, is smaller than the profit of each duopolist which is the profit of each firm if $p_{A}=p_{B}$, when $p_{A}=\frac{3 c}{2+3 c}$ the profit of the monopolist is equal to the profit of each duopolist, and when $p_{A}>\frac{3 c}{2+3 c}$ the profit of the monopolist is larger than the profit of each duopolist.

4. When $p_{A}<\frac{(3+\alpha) c}{2(1+\alpha)+(3+\alpha) c}$ we have

$$
\Pi_{A}^{M}<\Pi_{A}^{D},
$$

when $p_{A}=\frac{(3+\alpha) c}{2(1+\alpha)+(3+\alpha) c}$ we have

$$
\Pi_{A}^{M}=\Pi_{A}^{D},
$$

and when $p_{A}>\frac{(3+\alpha) c}{2(1+\alpha)+(3+\alpha) c}$, we have

$$
\Pi_{A}^{M}>\Pi_{A}^{D} .
$$

We can show

Also we can show

$$
\frac{c}{1+c}<\frac{(3+\alpha) c}{2(1+\alpha)+(3+\alpha) c}<\frac{3 c}{2+3 c} .
$$

and

$$
\text { when } \alpha \rightarrow 1, \frac{(3+\alpha) c}{2(1+\alpha)+(3+\alpha) c} \rightarrow \frac{c}{1+c}
$$


Let

$$
\text { when } \alpha \rightarrow 0 . \frac{(3+\alpha) c}{2(1+\alpha)+(3+\alpha) c} \rightarrow \frac{3 c}{2+3 c} \text {. }
$$

and differentiating $\bar{p}$ with respect to $\alpha$,

$$
\bar{p}=\frac{(3+\alpha) c}{2(1+\alpha)+(3+\alpha) c}
$$

$$
\frac{d \bar{p}}{d \alpha}=-\frac{4 c}{[2(1+\alpha)+(3+\alpha) c]^{2}}<0 .
$$

Therefore, the larger the value of $\alpha$ (the weight on the relative profit), the lower the value of $\bar{p}$.

\section{Absolute profit maximization}

In this section for reference we consider a case where each firm seeks to maximize its absolute profit. For details see Dastidar(1995).

First we show

\section{Lemma 1.}

There is no monopolistic equilibrium.

Proof. A monopolistic equilibrium is an equilibrium where Firm A is the monopolist. Suppose that $p_{A}<p_{B}$ and $p_{A}>\frac{c}{1+c}$. Then, Firm B can set $p_{B}$ slightly lower than $p_{A}$ and earn the positive profit. If $p_{A}<p_{B}$ and $p_{A}=\frac{c}{1+c}$, Firm A can set $p_{A}$ slightly higher than $\frac{c}{1+c}$ but lower than $p_{B}$ and earn the positive profit, or Firm B can set $p_{B}=p_{A}$ and earn the positive profit in duopoly. Of course $p_{A}<\frac{c}{1+c}$ is not profitable for Firm A. Q.E.D.

\section{Next we show}

\section{Theorem 1.}

There exists a range of the equilibrium prices $\frac{c}{2+c} \leq p \leq \frac{3 c}{2+3 c}$ in duopolistic equilibria.

Proof. A duopolistic equilibrium is an equilibrium where Firm A and B set the same price.

1. Suppose $p_{A}=p_{B}$ and $p_{A}>\frac{3 c}{2+3 c}$. Then, Firm B (or A) can set $p_{B}$ (or $p_{A}$ ) slightly lower then $p_{A}$ (or $p_{B}$ ), and increase its profit as a monopolist.

2. Suppose $p_{A}=p_{B}$ and $\frac{c}{2+c} \leq p_{A} \leq \frac{3 c}{2+3 c}$. Then, the profits of duopolists are non-negative. If Firm B (or A) sets $p_{B}$ (or $p_{A}$ ) lower than $p_{A}$ (or $p_{B}$ ), it becomes a monopolist but its profit decreases or does not change.

3. Of course if $p_{A}=p_{B}$ and $p_{A}<\frac{c}{2+c}$, the profits of the firms are negative, so each firm can set its price higher than the price of the rival firm and make its profit zero.

Q.E.D.

\section{Relative profit maximization}

In this section we consider a case where each firm seeks to maximize the weighted sum of its 
absolute profit and its relative profit.

First we show

\section{Lemma 2.}

There is no monopolistic equilibrium.

Proof. A monopolistic equilibrium is an equilibrium where Firm A is the monopolist. Suppose that $p_{A}<p_{B}$ and $p_{A}>\frac{c}{1+c}$. Then, Firm B can set $p_{B}$ slightly lower than $p_{A}$, and earn the positive absolute and relative profits. If $p_{A}<p_{B}$ and $p_{A}=\frac{c}{1+c}$, Firm A can set $p_{A}$ slightly higher than $\frac{c}{1+c}$ but lower than $p_{B}$ and earn the positive absolute and relative profits, or Firm $\mathrm{B}$ can set $p_{B}=p_{A}$, and earn the positive absolute profit in duopoly with zero relative profit because $\frac{c}{2+c}<\frac{c}{1+c}$. Of course $p_{A}<\frac{c}{1+c}$ is not profitable for Firm A. Q.E.D.

Next we show the existence of duopolistic equilibria.

\section{Theorem 2.} equilibria.

There exists a range of the equilibrium prices $\frac{c}{2+c} \leq p \leq \frac{(3+\alpha) c}{2(1+\alpha)+(3+\alpha) c}$ in duopolistic

Proof.

1. Suppose $p_{A}=p_{B}$ and $p_{A}>\frac{(3+\alpha) c}{2(1+\alpha)+(3+\alpha) c}$. The relative profits of the firms are zero. Firm B (or A) can set $p_{B}$ (or $p_{A}$ ) slightly lower then $p_{A}$ (or $p_{B}$ ), and earn the positive absolute profit as a monopolist. Although that profit is smaller than its profit in a duopolistic equilibrium when $p_{A}=p_{B}$ and $\frac{(3+\alpha) c}{2(1+\alpha)+(3+\alpha) c}<p_{A}<\frac{3 c}{2+3 c}$, its relative profit is positive and equal to its absolute profit because the profit of the rival firm is zero, and from (3) we have

$$
\Pi_{A}^{M}>\Pi_{A}^{D} \text {. }
$$

2. Suppose $p_{A}=p_{B}$ and $\frac{c}{2+c} \leq p_{A} \leq \frac{(3+\alpha) c}{2(1+\alpha)+(3+\alpha) c}$. Then, the absolute profits of duopolists are non-negative, and their relative profits are zero. If Firm $\mathrm{B}$ (or $\mathrm{A}$ ) sets $p_{B}$ (or $p_{A}$ ) lower than $p_{A}$ (or $p_{B}$ ), it becomes a monopolist, but in this case from (1) and (2) we have

$$
\Pi_{A}^{D} \geq \Pi_{A}^{M} \text {. }
$$

Thus, there is no incentive to deviate from the equilibrium.

3. Of course if $p_{A}=p_{B}$ and $p_{A}<\frac{c}{2+c}$, the absolute profits of the firms are negative and their relative profits are zero, so each firm can set its price higher than the price of the rival firm and make its absolute profit zero and its relative profit positive since the absolute profit of the rival firm is negative because $\frac{c}{2+c}<\frac{c}{1+c}$.

Q.E.D.

Note that the range of the equilibrium prices $\frac{c}{2+c} \leq p \leq \frac{(3+\alpha) c}{2(1+\alpha)+(3+\alpha) c}$ in duopolistic equilibria when the firms maximize the weighted sum of the absolute profits and the relative profits is contained in the range of the equilibrium prices $\frac{c}{2+c} \leq p \leq \frac{3 c}{2+3 c}$ in duopolistic equilibria under pure absolute profit maximization. The former is narrower and lower than the latter, and from (4) 
the larger the weight on the relative profit, $\alpha$, the narrower and lower the range of the equilibrium prices. Therefore, relative profit maximization is more aggressive than absolute profit maximization.

Acknowledgements. We are very grateful to the reviewers for their helpful comments on the original paper.

\section{References}

Dastidar, K.G. (1995) On the existence of pure strategy Bertrand equilibrium, Economic Theory, 5, 19-32.

Gibbons, R. and Murphy, K.J. (1990) Relative performance evaluation for chief executive officers, Industrial and Labor Relations Review, 43, 30S-51S.

Kockesen, L., Ok, E.A. and Sethi, R. (2000) The strategic advantage of negatively interdependent preferences, Journal of Economic Theory, 92, 274-299.

$\mathrm{Lu}, \mathrm{Y}$. (2011) The relative-profit-maximization objective of private firms and endogenous timing in a mixed oligopoly, The Singapore Economic Review, 56, 203-213.

Lundgren, C. (1996) Using relative profit incentives to prevent collusion, Review of Industrial Organization, $11,533-550$.

Matsumura, T., Matsushima, N. and Cato, S. (2013) Competitiveness and R\&D competition revisited, Economic Modelling, 31, 541-547.

Schaffer, M.E. (1989) Are profit maximizers the best survivors?, Journal of Economic Behavior and Organization, 12, 29-35.

Tanaka, Y. (2013) Irrelevance of the choice of strategic variables in duopoly under relative profit maximization, Economics and Business Letters, 2(2), 75-83.

Vega-Redondo, F. (1997) The evolution of Walrasian behavior, Econometrica, 65, 375-384. 